Mazzetti CH, Serinaldi F, Lebrun E, Lemaitre J.

Early laparoscopic adhesiolysis for small bowel obstruction: retrospective study of main advantages.

Surgical Endoscopy 2018

DOI: https://doi.org/10.1007/s00464-017-5979-x

\section{Copyright:}

The final publication is available at Springer via https://doi.org/10.1007/s00464-017-5979-x

Date deposited:

$16 / 02 / 2018$

Embargo release date:

07 December 2018

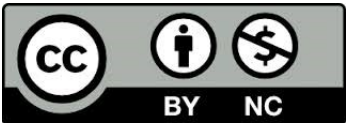

This work is licensed under a Creative Commons Attribution-NonCommercial 3.0 Unported License 


\title{
Early laparoscopic adhesiolysis for small bowel obstruction: retrospective study of main advantages
}

\author{
Claudia Hannele Mazzetti ${ }^{1}$, Francesco Serinaldi²,3, Eric Lebrun ${ }^{1}$, Jean Lemaitre' \\ ${ }^{1}$ Department of Visceral Surgery, Centre Hospitalier Universitaire Ambroise Pare, Mons, Belgium. \\ ${ }^{2}$ School of Civil Engineering and Geosciences, Newcastle University, Newcastle Upon Tyne, UK. \\ ${ }^{3}$ Willis Research Network, London, UK.
}

Key words: small bowel obstruction, laparoscopic adhesiolysis, postoperative adherence formation, management of small bowel adherences, early adhesiolysis, obstruction recurrences.

Corresponding author: Claudia Hannele Mazzetti, MD, Department of Visceral Surgery, Centre Hospitalier Universitaire Ambroise Pare, Bd Kennedy 2, 7000 Mons, Belgium claudiahannelemazzetti@gmail.com

\begin{abstract}
Background: The problem of managing adhesional small bowel obstruction (ASBO) is still unsolved. A conservative medical attitude is privileged even if it is associated to a high rate of recurrences, while surgery is applied to cases showing no improvement after 48-72 hours. Adhesiolysis via laparotomy has been the standard surgical management, but it causes other adhesions in a vicious circle. The aim of the study is to evaluate the advantages of early laparoscopic adhesiolysis as an alternative approach.

Methods: From January 2010 to April 2017, 107 patients were admitted with a diagnosis of ASBO. Patients underwent medical treatment, early surgery, emergency surgery or delayed surgery after failure of medical treatment. A retrospective review and explorative statistical analysis were
\end{abstract}


performed using graphical diagnostic plots, Mann-Whitney (MW) test, Kolmogorov-Smirnov (KS) test, exact binomial test, and $\chi^{2}$ test.

Results: Medical treatment led to resolution in the $77.3 \%$ of cases, but patients exhibit much more recurrences than those in the surgical group $\left(\chi^{2} p<.001\right)$. They also show a longer fasting time (MW $p=.027$; KS $p=.102$ ), a doubled number of radiological exams (MW $p<.001 ; \mathrm{KS} p<$. 001), and more major complications than those in the early surgery group. Early surgery group is associated to shorter fasting time (MW $p<.001$; KS $p<.001$ ), much shorter hospital stay (MW $p<$ .001 ; KS $p=.002$ ) and a smaller number of radiological exams (MW $p=.005 ; \mathrm{KS} p=.002$ ) compared with delayed surgery group. The laparoscopic group shows significantly earlier regain of intestinal transit (MW $p<.001 ; \mathrm{KS} p=.002$ ), shorter fasting time (MW $p=.002 ; \mathrm{KS} p=.008$ ), reduced number of radiological exams (MW $p=.003$; KS $p=.014$ ), reduced hospital stay (MW $p<$ $.001 ; \mathrm{KS} p=.005)$, and no more complications than the open surgery group.

Conclusions: Early laparoscopic surgery can be proposed as an effective alternative treatment for ASBO.

\section{Introduction}

\section{Adherences formation}

Adherences are defined as band of fibrotic tissue (scars) connecting surfaces that usually are not in contact. Their development is a major complication of surgery and it affects about the $93 \%$ of patients who underwent pelvic-abdominal surgery. Although the majority of these adherences remains silent and does not provoke any clinical symptoms, some of them can be responsible of "adhesive disease", a symptomatic state ranging from chronic abdominal pain (or infertility) to complete intestinal obstruction. 
Adhesiogenesis starts early during the surgery, as every insult on peritoneal sheet causes phlogosis that evolves in fibrosis. In the damaged area mast cells release histamine, which increases vascular permeability and attracts inflammatory cells. A fibrin gel matrix deposits, and macrophages direct mesothelial cells on the hurt area to reconstruct the lining. Riepithelialisation takes about 5-7 days. If the interrupted surfaces stay in contact they develop an adherence. This can happen between two or more intra-abdominal organs and/or the inner abdominal wall $[1,2,3]$.

\section{Causes of adherences}

Common causes of peritoneal insult can be classified in three general categories:

- Post surgical; more of the $90 \%$ resulting from prior abdominal and pelvic surgery, primarily laparotomy.

- Infections or inflammatory disease (appendicitis, diverticulitis, endometriosis, pelvic inflammatory disease, bowel intestinal disease, abdominal tubercolosis), chemical peritonitis (contact with bile, opening of dermoid cysts), and irritation from foreign body.

- Post radiation; radiation therapy used for treatment of multiples malignant disease, such as colorectal, prostatic, gynecologic cancer or limphoproliferative neoplasia, can cause early or late adhesions as a consequence of chronic ischemia of peritoneum. The severity is directly correlated to extent of the area treated, the dose of fractionation, and the total dose of radiation [4].

\section{Small bowel obstruction due to adherences}

Given the fibrotic nature of adhesive bands, they interfere with the regular intestinal motility producing, in the best case, abdominal discomfort, chronic bloating, cramping, alteration of bowel habits and constipation, nausea or early sensation of satiety. When extrinsic compression becomes 
complete, patients develop acute small bowel obstruction, whose pathognomonic signs are incoercible vomiting associate to cessation of gas and stools. Adhesional small bowel obstruction (ASBO) occurs in the $3 \%$ of all laparotomies, with the $1 \%$ occurring in the first postoperative year $[5]$.

\section{History of physiopathology of obstruction}

The understanding of the physiopathology of small bowel obstructions is mainly due to Owen Wangensteen, Chairman of the Department of Surgery at the University of Minnesota from 1930 to 1968. His experiments on dogs established the cause of signs and symptoms and their treatment. The experiments consisted of tying the mid-ileum, and then dividing the esophagus and bringing out the upper end to the skin as a mucous fistula in half of the animals. In the other half, the gastrointestinal tract was maintained intact. The first group did not become distended with either gas or fluid and survived for prolonged periods. The second one experienced the classical consequences of small bowel obstruction, until death. In this way, Wangensteen proved that: (i) swallowed air causes distention; (ii) the excess fluid accumulation above the obstruction is due to the pressure of the swallowed air on the bowel wall impeding venous outflow but not arteriolar inflow; and (iii) removing the air by means of gastric tubes (named Wangensteen Suction after him) improved clinical condition of both experimental animals and patients. Nasogastric suction allowed a safer surgery or even resolution without intervention in many cases.

Wangensteen established five main criteria that still apply today in order to consider the obstruction resolved, thus allowing the withdrawal of the suction: (i) cessation of "gas pains"; (ii) decrease of abdominal distention; (iii) visualization of gas in the colon on the radiograph; (iv) less fluid aspirated through the tube; and (v) toleration of temporary discontinuation of suction without recurrence of pain $[6,7]$. 


\section{Management of small bowel obstruction}

Any treatment for small bowel obstruction starts with medical management involving intravenous hydration, correction of electrolyte abnormalities, intravenous antibiotics, nil per os, and nasoenteral suction.

Laboratory tests measuring white blood cell count, C-reactive protein (CRP), blood urea nitrogen and creatinine, can assess the level of systemic illness. However, these clinical tests are not specific, whereas Lactate dehydrogenase $(\mathrm{LDH})$ is an abundant enzyme in intestinal mucosa that is considered a sensitive marker of bowel ischemia. In fact, when blood supply to bowel is compromised, mucosa suffers and serum levels of LDH increase. A recent study found that LDH > $1000 \mathrm{IU} / 1$ indicates gangrenous changes [8]. Erect plain radiography frequently shows multiple air fluid levels and distention of small bowel associated to absence of gas in the colon. The best radiological exams remains the abdominal $\mathrm{CT}$ scan, especially with administration of oral or intravenous contrast. It shows the transition point, identifying the cause of obstruction and possible complications such as perforation, ischemia or necrosis $[9,10]$.

The use of Gastrografin, the most common contrast medium, has been investigated as a method to stimulate the recovery of intestinal transit. In fact, its hyperosmolar power $(2150 \mathrm{mOsm} / \mathrm{l})$ activates movement of water into the small bowel lumen, decreases edema of the intestinal wall, and enhances smooth muscle contractile activity that can generate effective peristalsis. Presence of contrast in the colon is predictive of resolution.

A medical treatment can be successful in the $41 \%$ to $80 \%$ of cases $[11,12]$. There is no consensus about when a conservative treatment should be considered unsuccessful and the patient should undergo surgical adhesyolisis, but the World Society of Emergency Surgery 2013 established that 
non-operative management can be prolonged up to 72 hours if patients remain stable and there is no strangulation or peritonitis. After 72 hours, surgery is recommended [13,14,15,16,17].

The problem is to identify which patients will respond to conservative treatment and those who will need surgery, bearing in mind that a necessary but delayed surgery exposes the patient to a higher risk of intestinal resection. In fact, the overall mortality is $10 \%$ but it increases to $30 \%$ with bowel necrosis/perforation. Predicting factors for emergency surgery are the presence of free intraperitoneal fluid, mesenteric edema, small bowel feces sign at CT scan, involuntary abdominal guarding, and severe abdominal pain [14,18].

\section{Surgical treatment}

Open laparotomy has been considered for a long time the only possible surgical approach. However, laparotomy creates new adhesions and becomes itself the cause of recurrence. Laparoscopic adhesiolysis is emerging as an alternative to open surgery. In the past, it was informally banned in case of intestinal obstruction because of the complexity of surgery. In fact, coelioscopy can be challenging for the surgeon as the bowel distention reduces the visual field, makes the movements within the peritoneal cavity more difficult, and the intestinal wall more fragile and at greater risk of iatrogenic perforation. The first laparoscopic adhesiolysis in small bowel obstruction was performed by Mouret in 1972 [19]. Then, it was suggested for selected group of patients in case of first episode of obstruction or for patients with isolated bands. Today, a majority of surgeons accepts laparoscopy as initial step of exploration of the abdomen in case of bowel obstruction. A recent consensus conference established that the only absolute contraindications to laparoscopy for adhesiolysis are those related to pnemoperitoneum (hemodynamic instability or cardiopulmonary impairment) [20]. 
The surgery involves the use of Hasson's technique for open laparoscopy and placement of the first trocart in order to avoid accidental perforation of distended intestine that can be adherent to the anterior abdominal wall. Normally two $5 \mathrm{~mm}$ trocarts are sufficient to explore peritoneal cavity and they are placed under direct vision, respecting triangulation. The possibility of moving the operating table in different positions is very helpful to perform adhesyolisis since gravity removes the intestinal limbs from the visual field. Reported causes of laparotomic conversion are: (i) extended fibrous adhesions, especially in patients who have undergone more than two laparotomies; (ii) huge small bowel dilatation with a diameter of more than $4 \mathrm{~cm}$; (iii) presence of intestinal necrosis and consequent need for intestinal resection; and (iv) inadvertent enterotomy caused by inexperienced surgeon $[21,22,23,24,25]$.

When laparoscopic adhesiolysis is successfully accomplished, it seems to have clinically proven advantage over open approach as it results in less postoperative pain, faster regain of intestinal function and faster postoperative first meal, shorter hospital stay, decreased complications, lower healthcare costs, and decreased postoperative adhesions formation [26,27,28,29,30].

The current study resumes the experience of a peripheral surgical center. The aim is to investigate the outcome of the laparoscopic approach for small bowel obstructions and to compare conservative treatment versus surgical treatment in terms of short-term and long-term results.

\section{Materials and Methods}

From January 2010 to April 2017, 187 patients were admitted from Emergency Room to our department with a diagnosis of small bowel obstruction. Among these, 107 with adhesive causes were included after excluding other etiologies. Some patients were readmitted in different period with the same diagnosis, resulting in 125 hospitalizations. Causes of exclusion were pregnancy, age ( $<16$ years), peritoneal carcinomatosis, Crohn disease, other causes of mechanical obstruction (e.g., 
neoplasia, bezoars, foreign body, strangulated hernia), or causes of functional obstruction (e.g., Olgive syndrome and paralytic ileus). Data regarding demography, previous medical treatments, and follow-up were collected with a standardized data collection form.

Critically ill patients, as those who presented signs of gravity at $\mathrm{Ct}$ scan (abundant free peritoneal fluid, small bowel feces sign, mesenteric edema) or signs of peritoneal irritation, (involuntary abdominal guarding) were addressed directly to emergency laparotomy (ES). All the others underwent medical treatment (MT) or surgical treatment in the first 24 hours (S24) based on their clinical conditions, patient's will, surgeon's experience and preference, and complexity of the procedure. Our department routinely use Gastrografin for diagnostic and therapeutic purposes. When MT failed (no transit regain after 24 hours and until 72 hours or no contrast progression in the colon after 24 hours from the administration) patients were addressed to delayed surgery (DS) .

We retrospectively analyzed the following parameters: demographic features (age, gender, and BMI), number and type of previous surgical interventions, time between the last surgery and the present episode of obstruction, number of previous occlusion episodes, number of operated occlusions in the past, CRP and LDH values at the admission, type of treatment (medical and surgical), time of surgical treatment (ES, S24, and DS), type of surgical treatment (open adhesiolysis (OA) and laparoscopic adhesiolysis (LA)), number of intestinal resection cases, duration of hospital stay, time to regain intestinal function, total fasting time, number of radiological examinations, number of recurrences after treatment and minor/major complications.

The regain of intestinal function is identified by the presence of stools or gas associated to contrast in the colon and/or removal of nasogastric tube without vomiting. The fasting time is defined as the interval between the admission date to hospital and that of the first meal (even liquid) by mouth. 
The categorical data were compared using the $\chi^{2}$ test and the exact binomial test. Continuous variables were compared using the Mann-Whitney (MW) test and the two-sample KolmogorovSmirnov (KS) test along with graphical diagnostic plots of the empirical cumulative distribution functions (ECDFs). The statistical analyses were performed using $\mathrm{R}$ software [32]. P-values, $p$, smaller than 0.05 were considered as indication of statistical significance, bearing in mind the limits of null hypothesis statistical testing in ex post nonrandomized studies.

\section{Results}

The sample comprises 125 consecutive ASBO patients. Seventy five patients $(60 \%)$ underwent MT, $20(6 \%)$ were treated by S24, $8(6.4 \%)$ needed ES, and $22(17.6 \%)$ underwent DS after MT failure. There were no cases of death. Mean follow-up is 46.75 months, ranging within $(2,88)$ months. The age ranges in $(23,95)$ years with median value of 50.5 years. The population comprises 107 (distinct) patients: 62 (57.9\%) females, 44 (41.1\%) males, and $1(0.9 \%)$ transgender. Excluding the transgender patient, an exact binomial test yields $p=.098$, while the $95 \%$ confidence intervals (CIs) around the percentage of females in the 106 entries $(58.4 \%)$ is $(48.5,68.0)$. These results indicate that the adherences affect similar proportions of females and males.

BMI values range within $(14.87,37.89)$ for females, and $(14.69,40.04)$ for males, with median values of 24.38 and 26.59 for females and males, respectively. Empirical cumulative distribution functions (ECDFs) in Fig. 1 show that the BMI distribution of females tends to be shifted on the right compared to that of males, thus denoting systematic higher values for the latter group. Furthermore, only the $\sim 40 \%$ of females and $28 \%$ of males show an ideal weight, less than the $16 \%$ and $8 \%$ are respectively underweight, and the majority (44\% of females and $63 \%$ of males) are overweight, reflecting the known increase of BMI in the western population. 
Figure $2 \mathrm{a}$ shows that the most frequent previous surgical interventions were appendicectomy (28 patients, 27\%), laparotomy for different causes (predominantly abdominal wall surgery; 26 patients, $26 \%$ ), colectomy (24 patients, 23\%), and hysterectomy (24 patients, $22 \%$ ). Stratifying by gender (Fig. 2b), there is a high prevalence of hysterectomy in females (24 patients, 54.5\%). These data are in agreement with the current literature identifying multiple laparotomy, colorectal and pelvic surgery as risk factors in adherence formation $[12,31]$.

The $91.5 \%$ of patients had previous abdominal surgery; one or two previous surgeries account for the $73.6 \%$ of cases, while the probability to have patients with more than 2 previous surgeries is $17.9 \%$. Only 9 patients $(8.5 \%)$ did not undergo previous surgical procedures. Stratifying by gender, males generally comes with less previous surgeries $(77.3 \%$ with one or no surgeries against the $48.4 \%$ of females, and no cases with more than 4 previous operations).

The highest frequencies of obstruction cases occur within the first year and between 1 and 4 years from the most recent surgery too. It seems that females have a higher risk to suffer an occlusion between 1 and 4 years from the most recent surgery. For males, the risk is more spread across the time line, excluding the peak at time intervals less than 1 year.

Focusing on the S24 group (i.e., patients in which surgery was accomplished in the first 24 hours, excluding the cases of ES), and MT+DS group (i.e., all the patients initially addressed to conservative treatment, including patients who needed a delayed surgery at a second stage), their statistics are compared in Table 1 . We report median values and ranges in parentheses for continuous variables, and number of cases and corresponding percentages for categorical variables. The MT+DS group shows twice the number of radiological exams (MW $p<.001 ; \mathrm{KS} p<.001$ ), and a prolonged fasting time (MW $p=.027$ KS $p=.102$ ) with respect of the S24 group. In the $85 \%$ of cases there were no complications in both groups, but when complications happen, they tend to be major in the MT+DS group (9.3\% vs. 0\%). ECDFs in Figs. 3a-d provide a visual picture for the 
behavior of numeric variables. The two groups (S24 and MT+DS) behave similarly in the body of the distribution, while some discrepancies emerge in the upper part of the ECDFs. This behavior denotes that the two groups are similar on average, but the MT+DS group shows a higher probability to have unusually long fasting time, hospital stay, and regain of intestinal function.

We performed the same comparison between the S24 group and MT group, excluding patients of the DS group. Results are summarized in Table 2. The MT group requires twice the number of radiological exams of that of the $\mathrm{S} 24$ group (MW $p=.002$; KS $p<.001$ ), higher number of major complications $\left(\chi^{2} p=.050\right)$ and higher recurrence rate $\left(\chi^{2} p=.039\right)$. Of course, intestinal resection can only occur in the S24 group. ECDFs in Figs.3e-h confirm the results of the MW and KS tests. The S24 and MT groups show similar fasting time, hospital stay, and intestinal function regain. However, discrepancies in the upper part of ECDFs indicate that the medical treatment implies a higher probability to have unusually long hospital stay. In other words, the two treatments result in the same hospital stay on average; however, in some cases, the MT can require hospital stay much longer than that needed for S24. On the other hand, MT systematically needs a higher number of radiological exams, which can be very high in the most "complicated" cases.

MT was the initial approach to treat 97 patients. It led to resolution in 75 cases $(77.3 \%)$ and it failed in 22 patients $(22.6 \%)$, who then underwent DS.

Fifty patients out of 125 underwent surgical treatment. Of these 50 cases, 13 (26\%; 10 females and 3 males) were treated by laparoscopic adhesiolysis (LA), 3 (6\%; 1 female and 2 males) by LA subsequently converted in open adhesiolysis (OA) during the surgery when intestinal resection was required ( 2 cases) or the adhesion was too much extensive ( 1 case) and 34 (68\%; 23 females and 10 males) by OA. Focusing on the type of surgery, the comparison between the LA group (13 cases) and the OA group (34+3 conversions) is summarized in Table 3. The LA group shows statistically significant reduction of fasting time (MW $p=.002$; KS $p=.008$ ), shorter regain of 
intestinal function (KS $p=.002$ ) smaller number of radiological exams (MW $p=.003 ; \mathrm{KS} p=$. 014), shorter hospital stay (MW $p<.001$; KS $p=.005$ ) than those of the OA group. ECDFs in Figs. 3i-1 provide a visual display for the numeric variables and show a clear shift on the right (i.e., higher values) of the OA group with respect to the LA group for the considered parameters.

The comparison between the S24 group (20 patients) and DS group (22 patients) is summarized in Table 4. The former implies a median fasting time of 3 days with range $(1,7)$ while the latter 6 days ranging in $(4,20)(\mathrm{MW} p<.001 ; \mathrm{KS} p<.001)$. The difference between the times of regain of intestinal function is less evident, but the highest value for the DS group is more than twice the highest value of the S24 group. The DS group systematically requires more radiological exams (MW $p=.005 ; \mathrm{KS} p=.002$ ). There is no evident difference of type and frequency of complication, while the hospital stay is sensitively reduced for the S24 group (MW $p<.001$; KS $p=.002$ ). ECDFs in Figs. 4m-p confirm a systematic shift on the right (i.e., higher values are systematically more probable) of the DS group compared to the S24 group. For regain of intestinal function, the ECDFs of the two groups are similar, with discrepancies emerging in the extreme values. In other words, unlike the other parameters, the times to regain of intestinal function are generally similar, but the DS group can require very long times in some rare cases.

Finally, we compared the S24 group with ES group (Table 5). As one patient was operated twice in emergency, there 8 cases of ES and 7 distinct patients. All the cases were treated by OA. The comparison between the ES group and S24 group highlights that the former obviously shows a very prolonged fasting time (MW $p=.008$; KS $p=.032$ ), with the highest value (36 days) being five times greater than the highest value for the S24 group. The ES group has a higher time to regain normal intestinal function (MW $p<.001$; KS $p=.022$ ), higher number of radiological exams (MW $p=.005 ; \mathrm{KS} p=.022)$, and a higher risk of complications (75\% vs. $15 \%$ corresponding to S24 
Group; $\chi^{2} p<.001$ ), whereby the $62.5 \%$ of such complications are major. ECDFs in Figs. 3q-t confirm the differences between the characteristics of these two groups.

Median operative time was 57'(range 30'-125') for LA group; 64'(range 32'-155') for OA group and 96' (range 63'-126') for conversions, which was obviously influenced by necessity of intestinal resection. All operators included in this study were experienced general surgeons, accustomed to major advanced laparoscopic interventions (bariatric surgery, colo-rectal surgery). Trainees were always supervised by skilled surgeons.

LDH values range within $(104,929) \mathrm{UI} / 1$ for cases without resection, and $(162,430) \mathrm{UI} / 1$ for those with resection, with median values of 238 and 224 UI/1, respectively. ECDFs in Fig.4a show that the LDH distributions are similar in the two cases (light shaded areas denote the range $(125,250)$ $\mathrm{UI} / 1$ of normal values of $\mathrm{LDH}$ ). The MW and KS tests yield $p=.650$ and .828 , respectively (i.e., no evident difference).

CRP values span the range $(0.01,45) \mathrm{mg} / \mathrm{dl}$ for cases without resection, and $(0.09,15.9) \mathrm{mg} / \mathrm{dl}$ for cases with resection, with median values of 0.96 and $1.15 \mathrm{mg} / \mathrm{dl}$, respectively. ECDFs show that the CRP distributions are similar in the two cases (Fig. $4 \mathrm{~b}$; light shaded areas denote the range $(0,0.5)$ $\mathrm{mg} / \mathrm{dl}$ of normal values of CRP). MW and KS tests yield $p=.972$ and .903 , respectively (i.e., no evident difference).

By counting the relapses of hospitalizations in our department, recurrence rate was 1 out of 20 cases (5\%) for the S24 group (with 2 missing values), 1 out of 22 (4.5\%) for the DS group (with 1 missing values), 20 out of 75 (26.6\%) for the MT group (with 16 missing values), and 1 out of 8 (12.5\%) for the ES group (with 2 missing values). In order to investigate the actual number of relapses, we have included in our research also surgical or medical relapses affecting patients in previous hospitalizations. For each patient, we counted the number of occlusion episodes preceding the first admission (in our department), which were not treated by surgery. These cases are 
considered as failures of MT in as much admissions in other hospitals. Then, we counted the number of failures occurred in our admissions. Altogether, we have 29 MTs that failed in 29 previous hospitalizations and 20 failures in 75 MTs in our hospital. Therefore, there are 104 MTs: $49(47.1 \%)$ show relapses, $39(37.5 \%)$ do not, while information is not available for the remaining $16(15.4 \%)$ cases. In two cases, the readmission implied an ES with OA and intestinal resection.

Similar analysis was performed for surgical recurrences. For each patient, we counted the number of operations for occlusion preceding the first admission (in our department). These cases are considered as failures of surgical treatment in as much admissions in other hospitals. Then, we counted the number of failures of S24, DS, and ES occurred in the admissions in our department. Altogether, we have 4 surgical treatments that failed in 4 previous hospitalizations and 3 failures in 50 present surgical treatments. Therefore, there are 54 surgical treatments: 7 (13\%) show relapses, $42(77.8 \%)$ do not, while information is not available for the remaining $5(9.2 \%)$ cases.

Under the hypothesis that the surgical treatment yields less relapses than the medical one, missing values were treated in a conservative way favoring the falsification of such an assumption. Namely, all missing values were assumed to be "no relapses" in the case of MT, and "relapses" in the case of surgical treatment. This criterion yields 49 relapses out of $104(47.1 \%)$ for MT, and 12 out of 54 $(22.2 \%)$ for surgical treatment (see Table 6). The $\chi^{2}$ test yields $p=.002$ in favor of surgical treatment.

The lack of significant relationship between patients'ASA score and management or type of treatment (Table 7) indicates that no selection bias has occurred in our study. ASA 2 score is predominant for all groups, followed by ASA 3. Only one patient scored as ASA 4 was treated in the first 24 hours by open surgery.

\section{Discussion}


Formation of adherences, mainly due to abdominal surgery, is the first cause of small bowel obstruction. The problem of managing a first episode or even recurring episodes of intestinal occlusion is still unsolved. Currently, a conservative attitude, involving decompression of the intrabowel lumen by insertion of a suction tube and stimulation by administration of Gastrografin is often privileged, whereas surgical intervention is applied only to cases that do not show improvements after 48-72 hours. Even when MT is successful, it is associated with a high rate of recurrence and subsequent hospital admissions. Traditionally, laparotomy and adhesiolysis have been the standard surgical management, but OA often causes further adhesions in a vicious circle. Initially, laparoscopy for ASBO was informally banned because of greater difficulty, especially for untrained surgeons, and for suspected higher risk of iatrogenic injury compared with conventional laparotomy. Later, laparoscopy was limited to selected cases, while now it is more and more widespread as it is more frequently used for complex surgeries. Moreover, many studies have concluded that it is feasible and associated with early regain of intestinal transit, early refeeding, minor complications, and lower hospital stay.

This retrospective study reviews the cohort of ASBO patients admitted to our department and compare the features and outcomes of patients treated by medical vs. surgical treatment . The aim is to understand whether early laparoscopic surgery can be considered a better option to manage ASBO in terms of short-term results (hospital stay, fasting time and consequently time to refeeding, time to regain intestinal function, number of radiological exams during recovery), and mild-term results (minor and major complications, and rate of recurrence).

Results show that the patients initially addressed to medical treatment (MT and DS) have a reasonable probability of resolution $(77.3 \%$ of our cohort), but they show a prolonged fasting time, a doubled number of radiological exams, and more major complications in comparison with patients who underwent S24. If we exclude the DS group from the group initially treated by MT, we 
find that the difference in fasting time is no longer significant, as the fasting time of the DS group is obviously the sum of the time required by MT and the fasting time corresponding to peri- and postoperative phases. The time required for trying a conservative approach can depend on the operators who assess if the occlusion is resolved or not. Many centers established 72 hours, but early administration of Gastrograofin and a deadline of 24 hours could reduce this time. In our series patients received oral contrast pretty early but we do not assiste to immediate resolutions, attending sometimes more than 48 hours. On the other hand, when we exclude the DS group from the initial MT, we observe an increase of the rate of recurrence, thus making the difference between surgical and medical treatments significant in support of the better effectiveness of surgery in the long term.

The comparison between OA and LA highlights that the LA group systematically shows significantly earlier regain of intestinal transit ( 1 day on average with a maximum of 4 days vs. 4 days on average with a maximum of 24 days), shorter fasting time (only 3.5 days on average with a maximum of 5 days vs. 6 days on average with a highest value of 36), reduced number of radiological exams, reduced hospital stay and no more complications than those of the OA group. Therefore, LA for ASBO is not only less invasive and equally feasible than OA, but it has also positive implications in terms of costs and comfort for the patient.

Comparing the S24 and DS groups, the former is obviously associated to decreased fasting time. The differences in terms of regain of intestinal function are not significant, but S24 group shows shorter times of regain. The DS group systematically requires more radiological exams than the S24 group as the examinations to verify the effectiveness of the medical treatment must be added to the examinations for the surgical follow-up. Hospital stay is sensitively reduced in S24. To summarize, a surgery undertaken within the first 24 hours seems to have more positive effects than a DS. 
Finally, we analyzed the outcome of patients who underwent ES, since it comprises two patients who were treated several times only by MT. It should be highlighted that ES group is associated to a high risk of major complications and intestinal resection.

High LDH values and intestinal resection do not show any relationship. The highest values have been reported for patients who did not exhibit bowel necrosis and did not require resection. The same considerations hold true for CRP values. Since some patients were urgently taken out and suffered intestinal resection, in order to have comparable values of LDH and CRP (independently of the type of treatment), we used the values resulting from the clinical analysis performed at the time of admission. However, we believe that these parameters are not reliable enough and representative of the severity of the clinical situation. More likely, an analysis of the evolution of LDH and CRP values during the stay would provide a better picture, leading to other considerations and conclusions. Nonetheless, the low LDH values of the patients who underwent urgent resection for intestinal necrosis remain unexplained and deserve further research.

The Analysis of ASA score shows that the patient initial conditions do not influence the choice of the treatment, even though it should be noted that the majority of patients show mild systemic disease (ASA 2). However, we stress again that this study aims to review the standard treatment for the most patients rather than evaluating the best treatment for critical patients. Finally this study highlights advantages of early laparoscopic surgical approach to the ASBO problem: in hands of experimented surgeons laparoscopy is not more dangerous and when it is feasible shows better results, including comparable or sometimes best operative time. When conversion is necessary operative time is not too much incremented, with minimal risks for the patient. So we encourage always starts surgical treatment by laparoscopy.

The present study has some limitations: small sample, retrospective nature, selection bias due to non-randomization, and short follow-up time. If all patients underwent early laparoscopic 
surgery we cannot declare what the advantages or risk would be. Therefore, purposely designed perspective studies involving larger randomized samples and long follow-up period are required.

\section{Conclusions}

Early laparoscopic surgery can be proposed as an alternative of management for SBO due to adherences. Early surgery is associated with reduced number of radiological exams, minor complications and lower recurrence rate compared to medical treatment, while laparoscopic surgery is associated with earlier regain of intestinal function, decreased fasting time, shorter hospital stay, reduced number of radiological exams, minor complications compared to open surgery. Medical treatment can be successful in the majority of the cases but it is associated to high recurrence rate, which may end in surgery, even urgent. All possibilities should be discussed with the patients accounting for their comorbidities, histories of complex laparotomies (e.g., Bricker), and will. Further studies should be undertaken to demonstrate risk factors and to identify which patients may benefit from medical treatment and who will need later surgery in any case.

\section{Compliance with ethical standards}

Disclosures Claudia Hannele Mazzetti, Francesco Serinaldi, Eric Lebrun and Jean Lemaitre have no conflicts of interest or financial ties to disclose.

\section{References}

[1] Parker MC, Ellis H, Moran BJ, Thompson JN, Wilson MS, Menzies D, McGuire A, Lower AM, Hawthorn RJ, O’Briena F, et al. (2001) Postoperative adhesions: ten-year follow-up of 12,584 patients undergoing lower abdominal surgery. Dis Colon Rectum 44:822-829; discussion $829-830$ 
[2] Holmdahl L, Risberg B, Beck DE et al. (1997) Adhesions: pathogenesis and prevention-panel discussion and summary. Eur J Surg Suppl 557:56-62

[3] Di Zerega GS, Campeau JD. (2001) Peritoneal repair and post-surgical adhesion formation. Hum Reprod Update 7:547-555

[4] Tabibian N, Swehli E, Boyd A, Umbreen A, Tabibian JH (2017) Abdominal adhesions: A practical review of an often overlooked entity. Ann Med Surg (Lond) 15:9-13

[5] Duron JJ, Silva NJ, du Montcel ST, Berger A, Muscari F, Hennet H, Veyrieres M, Hay JM (2006) Adhesive postoperative small bowel obstruction: incidence and risk factors of recurrence after surgical treatment: a multicenter prospective study. Ann Surg 244:750-757

[6] Faryniuk A, MacDonald A, van Boxel P (2015) Amnesia in modern surgery: revisiting Wangensteen's landmark studies of small bowel obstruction. Can J Surg 58:83-84

[7] Edlich RF, Woods JA (1997) Wangensteen's transformation of the treatment of intestinal obstruction from empiric craft to scientific discipline. J Emerg Med 15:235-241

[8] O'Leary MP, Neville AL, Keeley JA, Kim DY, de Virgilio C, Plurad DS (2016) Predictors of Ischemic Bowel in Patients with Small Bowel Obstruction. Am Surg 82:992-994

[9] Obuz F, Terzi C, Sökmen S, Y1lmaz E, Y1ldız D, Füzün M (2003) The efficacy of helical CT in the diagnosis of small bowel obstruction. Eur J Radiol 48:299-304

[10] Zalcman M, Sy M, Donckier V, Closset J, Gansbeke DV (2000) Helical CT signs in the diagnosis of intestinal ischemia in small-bowel obstruction. Am J Roentgenol 75:1601-1607

[11] Selor D, Feign E, Szold A, Allweis TM, Carmon M, Nissan S, Freund HR (1993) How conservatively can postoperative small bowel obstruction be treated? Am J Surg 165:121-126

[12] Miron A, Giulea C, Nadragea M, Enciu O (2016) The Laparoscopic Approach of Small Bowel Obstruction--The Experience of a Primary Center. Chirurgia (Bucur) 111:126-130 
[13] Diaz Jr JJ, Bokhari F, Mowery NT, Acosta JA, Block EF, Bromberg WJ, Collier BR, Cullinane DC, Dwyer KM, Griffen MM, Mayberry JC (2008) Guidelines for management of small bowel obstruction. J Trauma Acute Care 64:1651-64

[14] Catena F, Di Saverio S, Coccolini F, Ansaloni L, De Simone B, Sartelli M, Van Goor H (2016) Adhesive small bowel adhesions obstruction: Evolutions in diagnosis, management and prevention. World J Gastrointest Surg. 8:222-231

[15] Di Saverio S, Coccolini F, Galati M, Smerieri N, Biffl WL, Ansaloni L, Tugnoli G, Velmahos GC, Sartelli M, Bendinelli C, et al. (2013) Bologna guidelines for diagnosis and management of adhesive small bowel obstruction (ASBO): 2013 update of the evidence-based guidelines from the world society of emergency surgery ASBO working group. World J Emerg Surg 8:42

[16] Leung $\mathrm{AM}, \mathrm{Vu} \mathrm{H}$ (2012) Factors predicting need for and delay in surgery in small bowel obstruction. Am Surg 78:403-407

[17] Catena F, Di Saverio S, Kelly MD, Biffl WL, Ansaloni L, Mandalà V, Velmahos GC, Sartelli M, Tugnoli G, Lupo M, et al. (2011) Bologna Guidelines for Diagnosis and Management of Adhesive Small Bowel Obstruction (ASBO): 2010 Evidence-Based Guidelines of the World Society of Emergency Surgery. World J Emerg Surg 6:5

[18] Otani K, Ishihara S, Nozawa H, Kawai K, Hata K, Kiyomatsu T, Tanaka T, Nishikawa T, Yasuda K, Sasaki K, Murono K, Watanabe T (2017) A retrospective study of laparoscopic surgery for small bowel obstruction. Ann Med Surg (Lond) 16:34-39

[19] Mouret P. L'adesiolisi coelioscopia. Chirurgia digestiva per via coelioscopica. (1994) In: Testa P, Delaitre B, editor. Edizioni Vigot, Friburgo; 53-69

[20] Vettoretto N, Carrara A, Corradi A, De Vivo G, Lazzaro L, Ricciardelli L, Agresta F, Amodio C, Bergamini C, Borzellino G, et al. (2012) Laparoscopic adhesiolysis: consensus conference guidelines. Colorectal Dis 14:e208-e215 
[21] Levard H, Boudet MJ, Msika S, Molkhou JM, Hay JM, Laborde Y, Gillet M, Fingerhut A (2011) Laparoscopic treatment of acute small bowel obstruction: a multicentre retrospective study. ANZ J Surg 71:641-646

[22] Suter M, Zermatten P, Halkic N, Martinet O, Bettschart V (2000) Laparoscopic management of mechanical small bowel obstruction: are there predictors of success or failure? Surg Endosc $14: 478-483$

[23] Grafen FC, Neuhaus V, Schob O, Turina M (2010) Management of acute small bowel obstruction from intestinal adhesions: indications for laparoscopic surgery in a community teaching hospital. Langenbecks Arch Surg 395:57-63.

[24] Ghosheh B, Salameh JR (2017) Laparoscopic approach to acute small bowel obstruction: review of 1061 cases. Surg Endosc 21:1945-1949

[25] Farinella E, Cirocchi R, La Mura F, Morelli U, Cattorini L, Delmonaco P, Migliaccio C, De Sol AA, Cozzaglio L, Sciannameo F (2009) Feasibility of laparoscopy for small bowel obstruction. World J Emerg Surg 4:3

[26] Pei KY, Asuzu D, Davis KA (2017) Will laparoscopic lysis of adhesions become the standard of care? Evaluating trends and outcomes in laparoscopic management of small-bowel obstruction using the American College of Surgeons National Surgical Quality Improvement Project Database. Surg Endosc 31:2180-2186

[27] Khalil OMH, Abdalla WM, Allam ZA (2016) Early laparoscopic adhesiolysis versus conservative treatment of recurrent adhesive small intestinal obstruction: a prospective randomized controlled trial. Egyptian J Surg 35:96-101

[28] Kelly KN, Iannuzzi JC, Rickles AS, Garimella V, Monson JR, Fleming FJ (2014) Laparotomy for small-bowel obstruction: first choice or last resort for adhesiolysis? A laparoscopic approach for small-bowel obstruction reduces 30-day complications. Surg Endosc 28:65-73 
[29] Nagle A, Ujiki M, Denham W, Murayama K (2004) Laparoscopic adhesiolysis for small bowel obstruction. Am J Surg 187(4):464-470

[30] Suh SW, Choi YS (2016) Laparoscopy for small bowel obstruction caused by single adhesive band. JSLS-J Soc Laparoend 20:e2016.00048

[31] Neff M, Schmidt B (2010) Laparoscopic treatment of a postoperative small bowel obstruction. JSLS-J Soc Laparoend 14:133-136

[32] R Development Core Team. R: A Language and Environment for Statistical Computing. Vienna, Austria, 2016. Available at: http://www.R-project.org/, Accessed January 6, 2017. 


\section{List of tables}

Table 1 : Comparison between S24 (early surgery) group and MT+DS group

Table 2 : Comparison between S24 group and MT group

Table 3 : Comparison between LA group and OA group

Table 4 : Comparison between S24 group and DS group

Table 5 : Comparison between S24 group and ES group

Table 6 : Comparison between MT and surgical relapses

Table 7: Contingency tables of ASA score versus management and type of treatment.

\section{List of figures}

Fig. 1: ECDFs of BMI for female and male population

Fig. 2: Frequency of previous surgical treatments stratified by typology (a), and typology and gender (b)

Fig. 3: Comparison of ECDFs of fasting time, time of intestinal function regain, number of radiological exams, and hospital stay for pairs of treatments: (a-d) Early surgery (S24) vs. Medical+Delayed (MT+DS), (e-h) Early surgery (S24) vs. Medical (MT+DS), (i-l) Laparoscopy (LA) vs. Open surgery (OA), (m-p) Early surgery (S24) vs. Delayed surgery (DS), and (q-t) Early surgery (S24) vs. Emergency surgery (ES)

Fig 4: ECDFs of LDH (a) and CRP values (b). Each panel compares ECDFs for cases with and without intestinal resection 
Table 1: Frequencies of different types of treatment

\begin{tabular}{lcccc}
\hline & Early Surgery (S24) & Emergency Surgery (ES) & Delayed Surgery (DS) & Medical (MT) \\
\hline Number of cases & 20 & 8 & 22 & 75 \\
\hline Percentage of cases & $16.0 \%$ & $6.4 \%$ & $17.6 \%$ & $60.0 \%$
\end{tabular}


Table 2: Frequencies of previous surgeries

Number of previous surgeries

\begin{tabular}{lcccccccc}
\hline & 0 & 1 & 2 & 3 & 4 & 5 & 6 & 7 \\
\hline Number of patients & 9 & 55 & 23 & 7 & 6 & 3 & 1 & 2 \\
\hline Percentage of patients & $8.5 \%$ & $51.9 \%$ & $21.7 \%$ & $6.6 \%$ & $5.7 \%$ & $2.8 \%$ & $0.9 \%$ & $1.9 \%$ \\
\hline
\end{tabular}


Table 3: Frequencies of previous surgeries accounting for gender stratification

\begin{tabular}{lcccccccc}
\hline & \multicolumn{7}{c}{ Number of previous surgeries } \\
\hline & 0 & 1 & 2 & 3 & 4 & 5 & 6 & 7 \\
\hline Number of female patients & 4 & 26 & 16 & 5 & 5 & 3 & 1 & 2 \\
\hline Percentage of female patients & $6.5 \%$ & $41.9 \%$ & $25.8 \%$ & $8.1 \%$ & $8.1 \%$ & $4.8 \%$ & $1.6 \%$ & $3.2 \%$ \\
\hline Number of male patients & 5 & 29 & 7 & 2 & 1 & - & - & - \\
\hline Percentage of male patients & $11.4 \%$ & $65.9 \%$ & $15.9 \%$ & $4.5 \%$ & $2.3 \%$ & - & - & - \\
\hline
\end{tabular}


Table 4: Comparison between S24 (early surgery) group and MT+DS group

\begin{tabular}{|c|c|c|c|c|c|c|}
\hline & & \multicolumn{2}{|c|}{ Treatment } & \multicolumn{3}{|c|}{ Tests } \\
\hline & & $\mathrm{S} 24$ & $\mathrm{MT}+\mathrm{DS}$ & MW $p$ & $\mathrm{KS} p$ & $\chi^{2} p$ \\
\hline Fasting time & & $3(1,7)$ & $3.5(1,20)$ & .027 & .102 & \\
\hline Intestinal transit regain (days) & & $2.5(1,6)$ & $2(0,15)$ & .431 & .606 & \\
\hline Number of radiological exams & & $2(1,9)$ & $4(1,53)$ & $<.001$ & $<.001$ & \\
\hline \multirow[t]{3}{*}{ Complications } & major & $0(0 \%)$ & $9(9.3 \%)$ & & & .122 \\
\hline & minor & $3(15 \%)$ & $5(5.2 \%)$ & & & \\
\hline & no & $17(85 \%)$ & $83(85.5 \%)$ & & & \\
\hline Hospital stay & & $7(4,16)$ & $7(3,45)$ & .464 & .596 & \\
\hline Intestinal resection (yes\%) & & $3(15 \%)$ & $4(4.1 \%)$ & & & .177 \\
\hline Recurrences (yes\%) & & $1(5 \%)[2 \mathrm{NAs}]$ & $21(21.6 \%)[17$ NAs $]$ & & & .112 \\
\hline
\end{tabular}


Table 5: Comparison between S24 group and MT group

\begin{tabular}{|c|c|c|c|c|c|c|}
\hline & & \multicolumn{2}{|c|}{ Treatment } & \multicolumn{3}{|c|}{ Tests } \\
\hline & & $\mathrm{S} 24$ & MT & MW $p$ & $\mathrm{KS} p$ & $\chi^{2} p$ \\
\hline Fasting time & & $3(1,7)$ & $3(1,11)$ & .187 & .248 & \\
\hline Intestinal transit regain (days) & & $2.5(1,6)$ & $2(0,6)$ & .578 & .817 & \\
\hline Number of radiological exams & & $2(1,9)$ & $4(1,53)$ & .002 & $<.001$ & \\
\hline \multirow[t]{3}{*}{ Complications } & major & $0(0 \%)$ & $5(6.7 \%)$ & & & .050 \\
\hline & minor & $3(15 \%)$ & $2(3.7 \%)$ & & & \\
\hline & no & $17(85 \%)$ & $68(90.6 \%)$ & & & \\
\hline Hospital stay & & $7(4,16)$ & $6(3,45)$ & .866 & .914 & \\
\hline Intestinal resection (yes\%) & & $3(15 \%)$ & $0(0 \%)$ [expected] & & & .007 \\
\hline Recurrences (yes\%) & & $1(5 \%)[2 \mathrm{NAs}]$ & $20(26.7 \%)[16 \mathrm{NAs}]$ & & & .039 \\
\hline
\end{tabular}


Table 6: Comparison between LA group and OA group

\begin{tabular}{|c|c|c|c|c|c|c|}
\hline & & \multicolumn{2}{|c|}{ Treatment } & \multicolumn{3}{|c|}{ Tests } \\
\hline & & LA & $\mathrm{OA}$ & $\mathrm{MW} p$ & $\mathrm{KS} p$ & $\chi^{2} p$ \\
\hline Fasting time & & $3.5(1,5)$ & $6(2,36)$ & .002 & .008 & \\
\hline Intestinal transit regain (days) & & $1(1,4)$ & $4(1,24)$ & $<.001$ & .002 & \\
\hline Number of radiological exams & & $2(1,5)$ & $5(1,41)$ & .003 & .014 & \\
\hline \multirow[t]{3}{*}{ Complications } & major & $1(7.7 \%)$ & $8(21.6 \%)$ & & & .081 \\
\hline & minor & $0(0 \%)$ & $7(18.9 \%)$ & & & \\
\hline & no & $12(92.3 \%)$ & $22(59.5 \%)$ & & & \\
\hline Hospital stay & & $6.5(4,17)$ & $10(4,55)$ & $<.001$ & .005 & \\
\hline Intestinal resection (yes $\%)$ & & $1(7.7 \%)$ & $10(27 \%)$ & & & .290 \\
\hline Recurrences (yes\%) & & 0 of $11(0 \%)$ [2 NAs] & 3 of $34(8.8 \%)$ [3 NAs] & & & .746 \\
\hline
\end{tabular}


Table 7: Comparison between S24 group and DS group

\begin{tabular}{|c|c|c|c|c|c|c|}
\hline & & \multicolumn{2}{|c|}{ Treatment } & \multicolumn{3}{|c|}{ Tests } \\
\hline & & $\mathrm{S} 24$ & DS & $\mathrm{MW} p$ & $\mathrm{KS} p$ & $\chi^{2} p$ \\
\hline Fasting time & & $3(1,7)$ & $6(4,20)$ & $<.001$ & $<.001$ & \\
\hline Intestinal transit regain (days) & & $2.5(1,6)$ & $3(1,15)$ & .123 & .210 & \\
\hline Number of radiological exams & & $2(1,9)$ & $5(2,41)$ & $<.001$ & .002 & \\
\hline \multirow[t]{3}{*}{ Complications } & major & $0(0 \%)$ & $4(18.2 \%)$ & & & .133 \\
\hline & minor & $3(15 \%)$ & $3(13.6 \%)$ & & & \\
\hline & no & $17(85 \%)$ & $15(68.2 \%)$ & & & \\
\hline Hospital stay & & $7(4,16)$ & $11(6,31)$ & $<.001$ & .002 & \\
\hline Intestinal resection (yes $\%$ ) & & $3(15 \%)$ & $4(18.2 \%)$ & & & $\sim 1$ \\
\hline Recurrences (yes\%) & & $1(5 \%)[2$ NAs $]$ & $1(4.5 \%)[1 \mathrm{NA}]$ & & & $\sim 1$ \\
\hline
\end{tabular}


Table 8: Comparison between S24 group and ES group

\begin{tabular}{|c|c|c|c|c|c|c|}
\hline & & \multicolumn{2}{|c|}{ Treatment } & \multicolumn{3}{|c|}{ Tests } \\
\hline & & S24 & $\mathrm{ES}$ & MW $p$ & $\mathrm{KS} p$ & $\chi^{2} p$ \\
\hline Fasting time & & $3(1,7)$ & $5.5(2,36)$ & .008 & .032 & \\
\hline Intestinal transit regain (days) & & $2.5(1,6)$ & $5.5(3,24)$ & $<.001$ & .022 & \\
\hline Number of radiological exams & & $2(1,9)$ & $10.5(1,19)$ & .005 & .022 & \\
\hline \multirow[t]{3}{*}{ Complications } & major & $0(0 \%)$ & $5(62.5 \%)$ & & & $<.001$ \\
\hline & minor & $3(15 \%)$ & $1(12.5 \%)$ & & & \\
\hline & no & $17(85 \%)$ & $2(25.0 \%)$ & & & \\
\hline Hospital stay & & $7(4,16)$ & $22(6,55)$ & .001 & .004 & \\
\hline Intestinal resection (yes $\%$ ) & & $3(15 \%)$ & $4(50 \%)$ & & & .140 \\
\hline Recurrences (yes\%) & & $1(5 \%)[2 \mathrm{NAs}]$ & $1(12.5 \%)[2 \mathrm{NAs}]$ & & & $\sim 1$ \\
\hline
\end{tabular}


Table 9: Comparison between MT and surgical relapses

\begin{tabular}{|c|c|c|c|c|c|}
\hline & \multicolumn{4}{|c|}{ Treatment } & \multirow{2}{*}{$\frac{\text { Test }}{\chi^{2} p}$} \\
\hline & MT (current) & MT (previous) & Surgical (current) & Surgical (previous) & \\
\hline Number of hospitalisations & 75 & 29 & 50 & 4 & \\
\hline Overall hospitalisations & \multicolumn{2}{|c|}{104} & \multicolumn{2}{|c|}{54} & \\
\hline Recurrences & $20(26.6 \%)$ & 29 & $3(6 \%)$ & 4 & $<.001$ \\
\hline Overall recurrences & \multicolumn{2}{|c|}{$49(47.1 \%)$} & \multicolumn{2}{|c|}{$7(13 \%)$} & \\
\hline N/A against our thesis & 16 & & 5 & & \\
\hline Overall recurrences w/ NAs & \multicolumn{2}{|c|}{$49(47.1 \%)$} & \multicolumn{2}{|c|}{$12(22.2 \%)$} & .002 \\
\hline
\end{tabular}

